\title{
XVII. Bauarbeiten in München und in der Nördlinger Druckerei \\ Ein vielseitig begabter Architekt: Roderich Fick
}

ls Heinrich Beck 1949 seinen Verlag wieder übernahm, sind es in
Nördlingen noch die alten Gebäude gewesen, in denen die Dru-
ckerei untergebracht war, nämlich seit 1775 die Adresse Bergerstraße 3 - das ursprüngliche Haus musste 1856 abgerissen und durch einen Neubau ersetzt werden - und die im selben Jahr dazugekommene Ergänzung des Druckhauses Bergerstraße 5. Der Wiederaufbau von Verlag und Druckerei nach der Währungsreform ist auch einer im wahrsten Sinne des Wortes gewesen, nämlich die Geschichte einer ständigen Bautätigkeit. Dazu meinte Heinrich Beck in seiner 1963 geschriebenen Verlagsgeschichte, die Beschäftigung mit Bauplänen sei für ihn «beinahe zu einem zweiten Beruf» geworden. Nicht nur in Nördlingen musste die Druckerei erweitert werden. Zuerst ist das am Ende des Kriegs zerstörte Verlagsgebäude wieder aufgebaut worden. Der Hauptbau wurde 1951 fertig. Mit seinem Anbau von $195^{2}$ scheint er für den heutigen Betrachter viel älter zu sein. Tatsächlich steht er mit seiner schönen konservativen Struktur unter Denkmalschutz. Das und die ähnlich sensible Anpassung der Druckerei an die mittelalterliche Umgebung der Altstadt in Nördlingen ist das Verdienst des Architekten Roderich Fick und seiner Frau Catherina, die nach seinem Tod auf der Grundlage von Planungen ihres Mannes die Arbeiten in Nördlingen weitergeleitet hat. Roderich Fick hatte ${ }_{1938}$ Heinrich Becks Wohnhaus am Biederstein im nördlichen Schwabing gebaut. Seit jener Zeit kannten sie sich. Wer beide Gebäude betrachtet, erkennt als Gemeinsamkeit Roderich Ficks charakteristischen Stil. Dieser zeigt sich in feinsinnig ausgewogenen Proportionen und in der gediegenen Gestaltung der Details.

Roderich Fick (1886-1955), geboren in Würzburg, aufgewachsen in Zürich, war außergewöhnlich vielseitig tätig und begabt. 1907 begann er ein Architekturstudium in München, Zürich und Dresden, das er ohne Abschlussexamen beendete, um lieber gleich in die Praxis zu gehen, bekam aber kaum Aufträge, hörte deshalb in Zürich Vorlesungen über Geologie, Meteorologie, Luftschifffahrt und Astronomie, beteiligte sich 1911 an einer Arktis-Expedition mit Ausrüstungsgegenständen, die er selbst hergestellt 


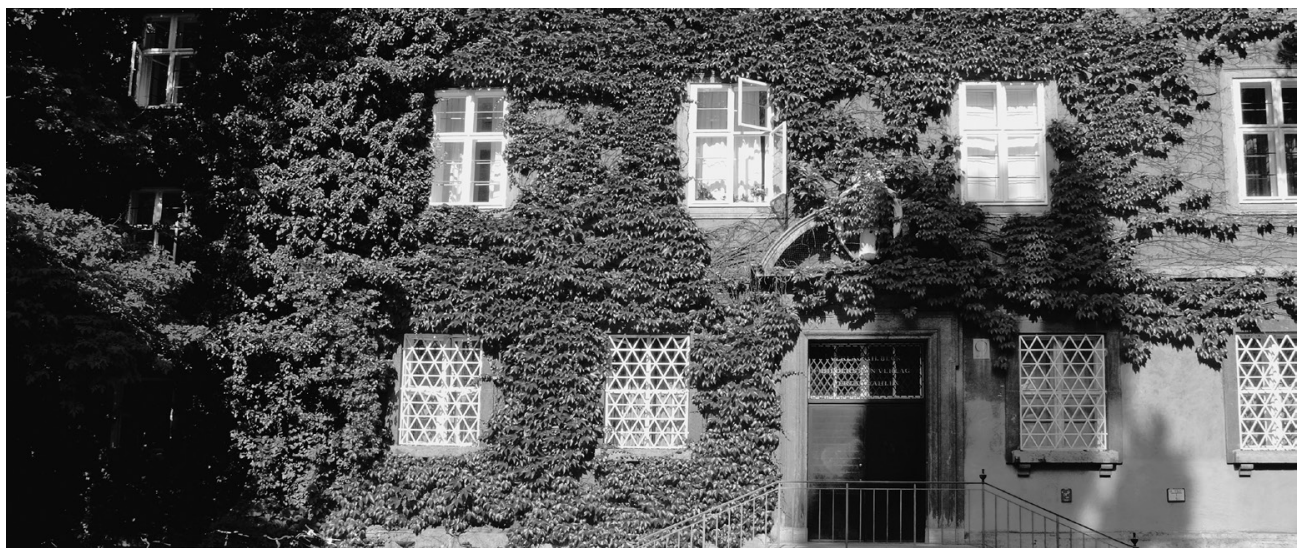

Roderich Fick gestaltete bis ins Detail: Eingang des Verlagsgebäudes in Schwabing, Wilhelmstr. 9 (der sog. Altbau, links anschließend der Anbau).

hatte, wurde 1914, Ingenieur in der deutschen Kolonie Kamerun, dort nach Ausbruch des Ersten Weltkriegs Offizier der Schutztruppe, die sich vor den Engländern auf spanisches Kolonialgebiet rettete und in Spanien interniert worden ist. Dort bildete er sich weiter, ging nach dem Krieg mit seinen Eltern und seiner ersten Frau nach Herrsching am Ammersee, wo er eine alte Wassermühle ausbaute und mit einer handwerklichen Firma, einer Bootswerft und der Konstruktion von Segelflugzeugen versuchte, sich eine neue Existenz aufzubauen. Schließlich erhielt er die ersten Aufträge als Architekt und wurde allmählich bekannt. 1935 kam der Durchbruch. Er hatte das «Haus der Deutschen Ärzte» in der Münchener Briennerstraße gebaut, das heute noch erhalten ist.

Dieses dreistöckige Gebäude ist typisch für seine Arbeit, ein eher schlichter Bau, der dort sensibel in die klassische Umgebung eingefügt wurde, ohne seine Herkunft aus der Gegenwart zu verleugnen. Man kann ihn als konservativ bezeichnen mit seinem großen ebenerdigen Eingangsportal, das umgeben ist von größeren Natursteinen. Das war jedenfalls eine klare Absage an die moderne Architektur des Bauhauses mit seinen Vertretern wie Mies van der Rohe oder Walter Gropius, deren äußere Form nur bestimmt wurde durch die innere Funktion unter Verzicht auf alles Überflüssige wie Stuck oder Ornamente. Dieses Gebäude der Ärzte war nicht weit entfernt vom «Braunen Haus», der Parteizentrale der NSDAP. Adolf Hitler ist bei der Einweihung dabei gewesen, bewunderte die Architektur und ließ sich ihren Urheber vorstellen. So kam Roderich Fick zu der fragwürdigen Ehre eines Gesprächs mit dem «Führer». Es hatte für ihn die 


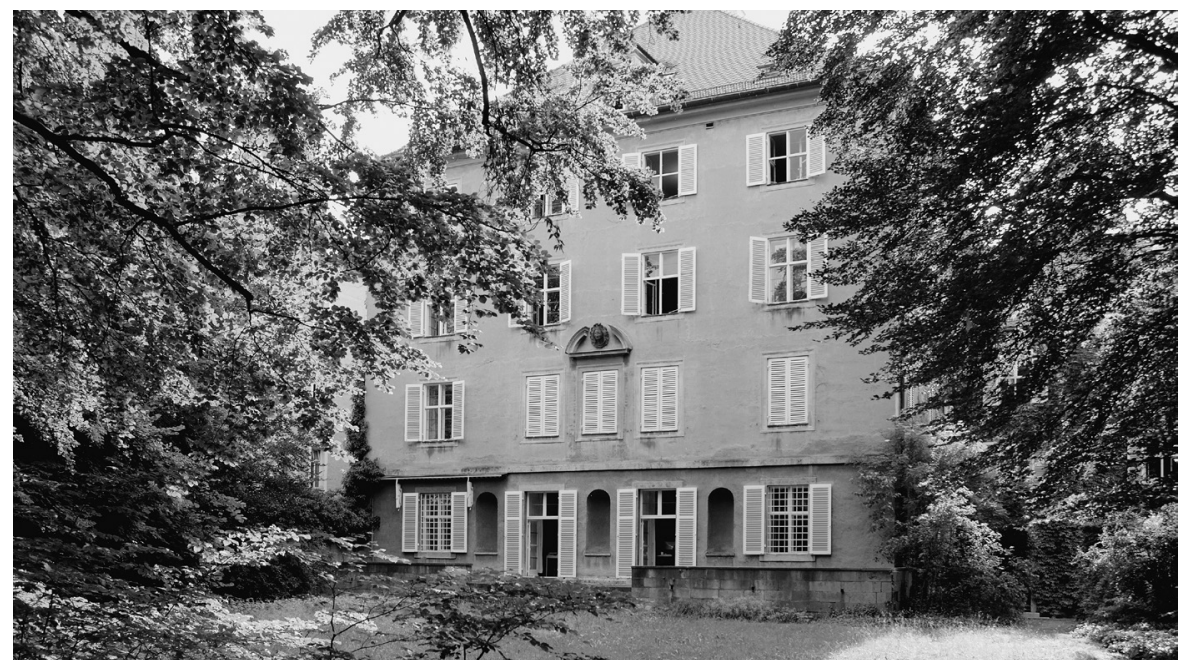

Verlagsgebäude in Schwabing von Roderich Fick, Wilhelmstr. 9 (der sog. Altbau) von der Gartenseite aus; rechts schließt der Anbau an.

angenehme Folge einer Flut von Aufträgen durch Partei und Staat, die ihn zu einem der wichtigsten Architekten im «Dritten Reich» machte. So hat er seit 1936 die meisten Gebäude am Obersalzberg gebaut, Hitlers Feriensitz in der majestätischen Bergwelt des bayerischen Berchtesgaden, so das heute noch existierende Kehlsteinhaus, das Hotel Patterhof, auch Zweckbauten wie Siedlungen für Bauarbeiter, Kasernen für die Bewacher des abgesperrten «Führergebiets» oder ein Verwaltungsgebäude für Hermann Göring und den Gutshof Martin Bormanns. Ungefähr vier Jahre hat er dort gearbeitet, wurde inzwischen Professor für Architektur an der Technischen Hochschule München und 1937 - wie Heinrich Beck - Mitglied der NSDAP. Nach Streitigkeiten mit Martin Bormann, Reichsleiter der NSDAP, hat er 1940 um Entlassung aus der Stellung des dort leitenden Architekten gebeten.

Er zog sich zurück auf seine Tätigkeit als vom «Führer» 1939 ernannter «Reichsbaurat» für die Stadt Linz, in der Hitler die schönsten Jahre seiner Jugend erlebt hat. Bis 194,1 hat er hier die Donaubrücke gebaut, das Hotel Donauhof und das Wasserstraßenamt. Dann kam er wieder durch Bormann unter Druck und konzentrierte sich nun auf seine Arbeit als Professor in München und private Bauten, war aber noch in der Endphase des Kriegs auf der damals von Hitler und Goebbels geführten Sonderliste von über tausend Künstlern, die dem Regime wichtig erschienen, um sie vom Dienst in der Wehrmacht zu befreien, offiziell als «Gottbegnadetenliste» 


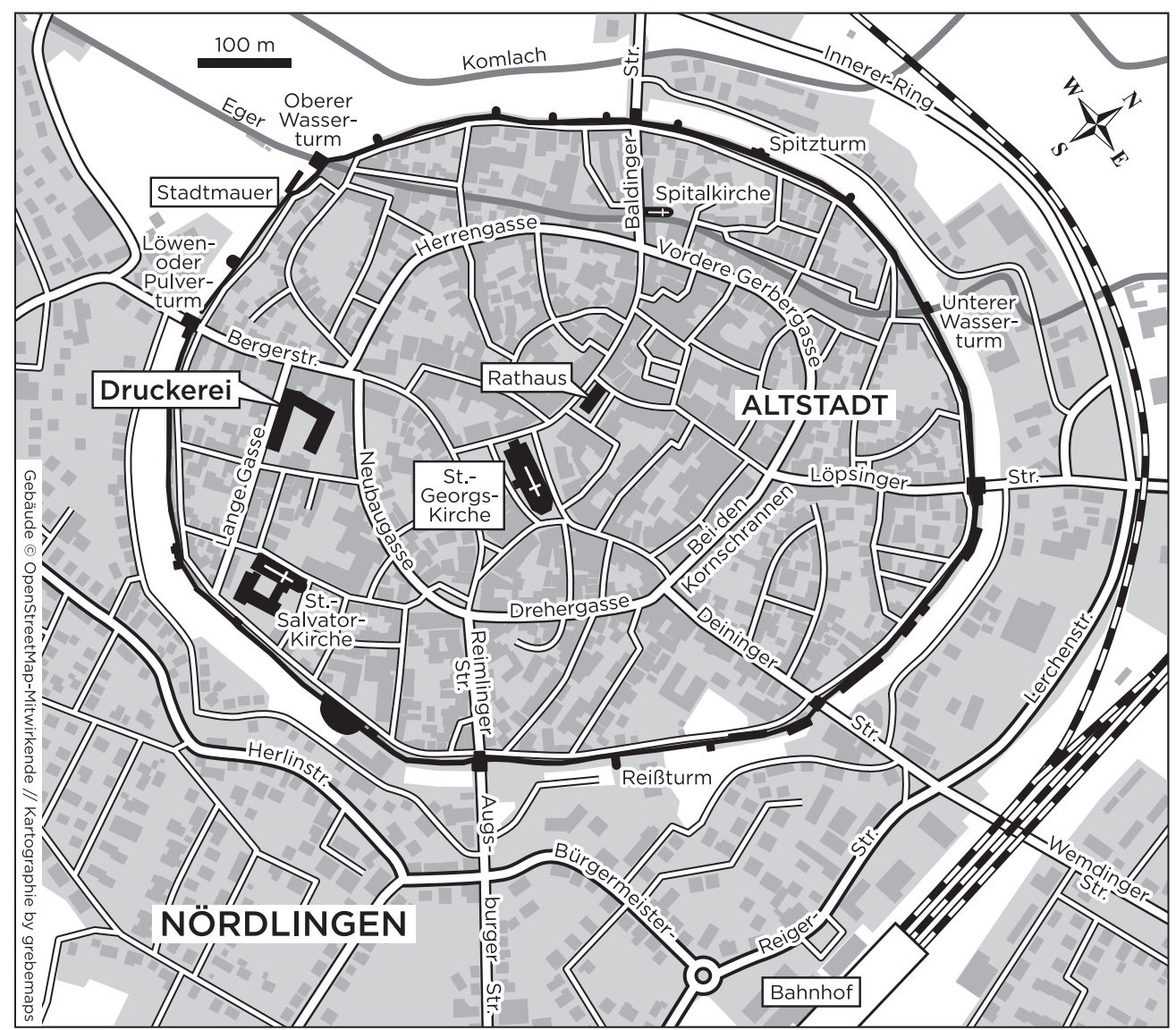

Die Altstadt von Nördlingen und die Druckereigebäude.

bezeichnet. Bis zum Ende dieser schlimmen Zeit hat er auch bei Aufträgen von Partei und Staat immer seinen eigenen Stil behalten, der schwer zu beschreiben ist, weil er ständig wechselte, je nach sensibler Einordnung in die Umgebung, aber immer in solider Handwerklichkeit und mit großer Liebe zum Detail. Nirgendwo hat er Konzessionen gemacht an den oft pompös-bombastischen Baustil der NS-Architektur und hat insgesamt gut verdient. Eine Million Reichsmark netto in den Jahren seit 1936, gab er an vor der Spruchkammer in München, und immer nur abgerechnet nach dem geringsten Satz der Honorarordnung für Architekten.

Nach Kriegsende wurde Roderich Fick wie Heinrich Beck wegen seiner Mitgliedschaft in der NSDAP und aktiver Tätigkeit für das «Dritte Reich» nach der JCS 1067 entlassen, er als Professor, Heinrich Beck als Verleger. Auch er wurde angeklagt nach dem Befreiungsgesetz vor derselben 
Spruchkammer X in München wie Heinrich Beck (siehe S. 189ff.). Für beide endete das Verfahren mit einem Urteil über die Einstufung als «Mitläufer» der Kategorie 4. Roderich Fick war nun nicht mehr der Lieblingsarchitekt Adolf Hitlers, sondern jetzt der von Heinrich Beck. Beide waren sich sehr ähnlich, vielleicht sogar befreundet. Auch ihm ist später zu Unrecht vorgeworfen worden, er habe aktiv nationalsozialistisch gehandelt. Richtig ist, dass beide vom «Dritten Reich» profitiert haben. Roderich Fick wurde einer der wichtigsten Architekten, Heinrich Beck einer der wichtigsten juristischen Verleger. Diese dunklen Flecke bleiben.

Nachdem die Bauarbeien in München mit dem Hauptbau/Altbau und dem Anbau in Richtung Ainmillerstraße fertig geworden waren, begannen Heinrich Beck und sein Architekt mit dem Ausbau der Nördlinger Druckerei. 1954, wurde rechtwinklig zur Bergerstraße ein erster Westflügel in der Langen Gasse fertig, 1967 dessen Fortsetzung bis zur Bräugasse und schon 1959 auch wieder im rechten Winkel zur Bergerstraße auf der anderen östlichen Seite an der Neubaugasse das «Versandgebäude», ebenfalls bis zur Bräugasse. Der Versand fand hier allerdings nur im Erdgeschoss statt, darüber wie in den Ergänzungsbauten der Langen Gasse waren Räume für Satz, Druck und Buchbinderei. So entstand zur Bräugasse ein Geviert, das ein wenig offen war für eine Einfahrt in den Hof, denn hier standen noch vier alte Häuser, die Heinrich Beck vorsichtshalber dazugekauft hatte. Eins musste bald abgerissen werden.

Danach folgte etwa ein Jahrzehnt Bauruhe in München und Nördlingen. Inzwischen wurden beide wieder größer, Verlag und Druckerei. Deshalb ging es seit 1978 weiter, hier wie dort. Das war nun schon die Initiative des jungen Verlegers Hans Dieter Beck. In München sind rechtwinklig zur Wilhelmstraße zwei Neubauten in der Ainmillerstraße entstanden, zuerst der «Neubau», später der «Erweiterungsbau». Doch dies soll später geschildert werden (siehe S. 314,f.).

Nun zur technischen Entwicklung der Druckerei bis zum Ende der aktiven Zeit Heinrich Becks. Nachdem man bis zum Anfang der Bundesrepublik in Nördlingen ständig damit beschäftigt war, die ausgeleierten Vorkriegsmaschinen mit intensiver Tüftelei und oft viel Zeit mühsam auszubessern, konnten seit 1950 neue Geräte angeschafft werden. Die erste große Wende für die Druckerei kam 1962, nachdem sie die Produktion sämtlicher dtv-Bände übernommen hatte. Deren erstes Taschenbuch, immer wieder aufgelegt, war Heinrich Bölls «Irisches Tagebuch». Nun kam die «Martini», eine in der Schweiz hergestellte Buchbindemaschine, die eine Fertigstraße war für die Druckbögen der Taschenbücher oder später 


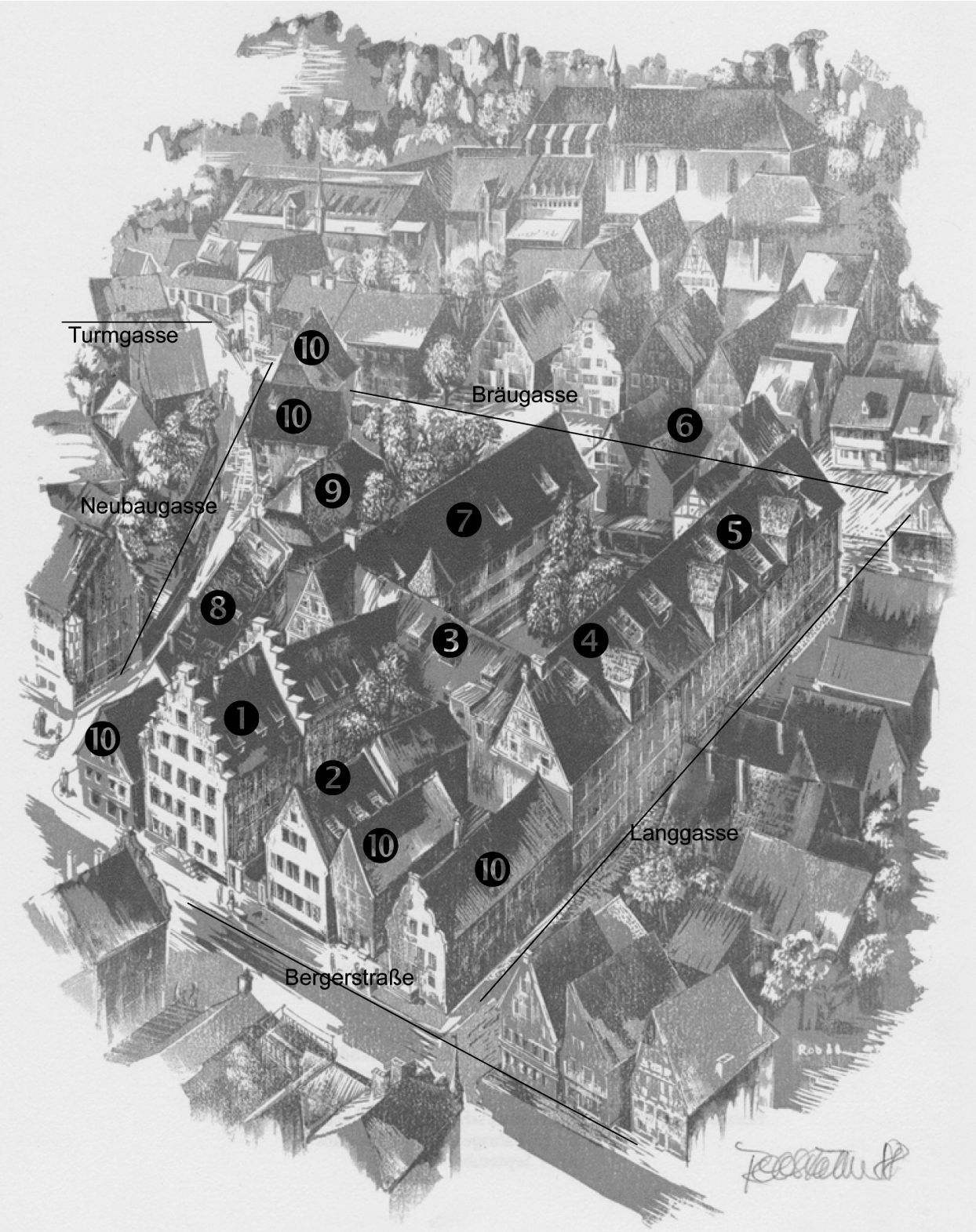
63 Beck'sche-Häuser verkauft an Lebenshilfe
(7) Versandgebäude 1959
8 Gerstmeierhaus, jetzt von Verkauf genutzt
(9) ehemaliges Werkstattgebäude/Fahrradraum
(11) Privatgebäude

Lageplan der Druckereigebäude in Nördlingen 


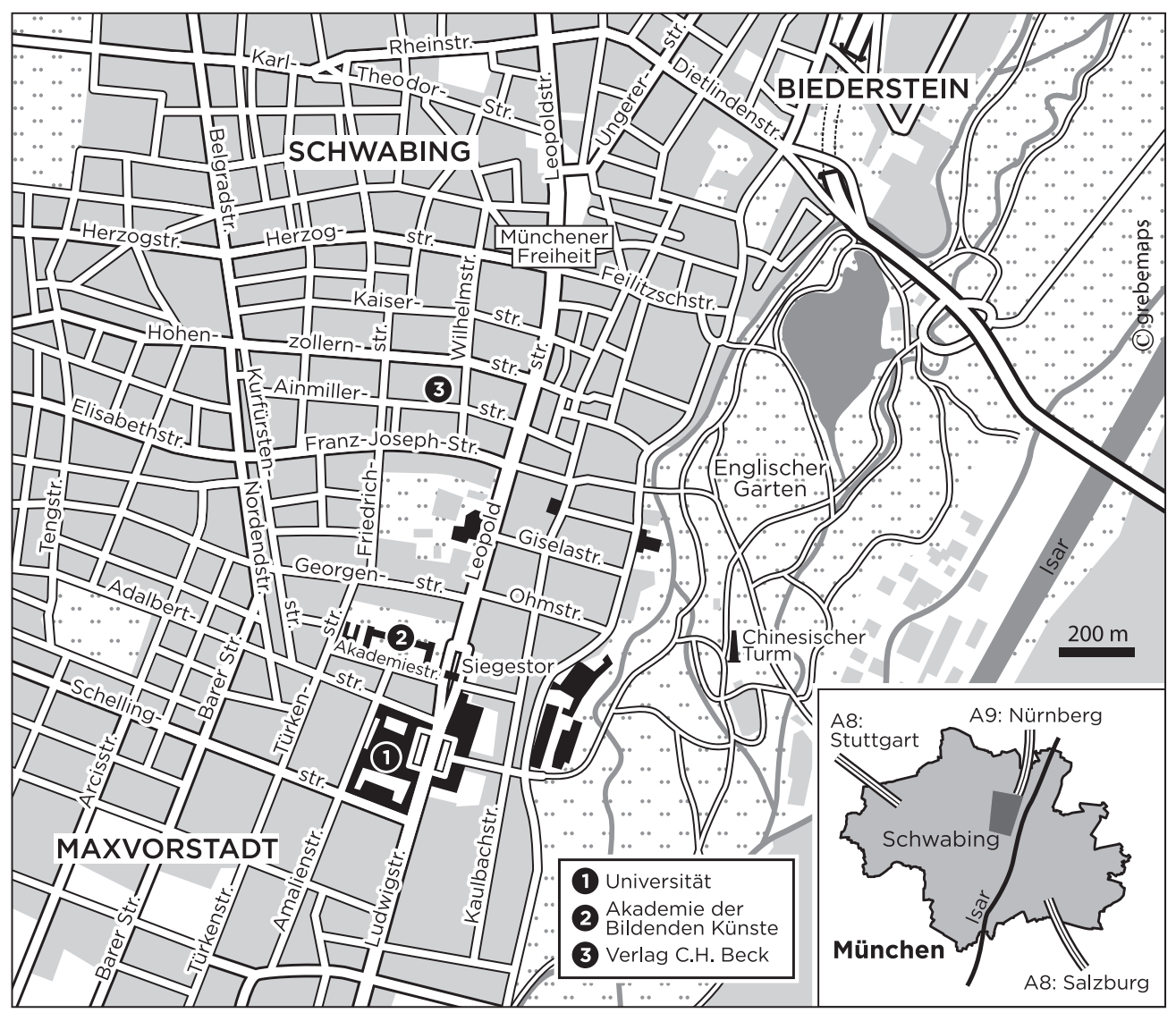

Lageplan des Verlags in München.

von Paperbacks, die sie am Ende als fertig gebundene Bände verließen. Zur selben Zeit fand die zweite Revolution der Buchherstellung in der Beck'schen Druckerei statt. Bleisatz und Buchdruck wurden abgelöst durch Fotosatz und Offsetdruck. Die waren leistungsstärker und es konnte billiger produziert werden.

In den fünfziger Jahren hatte man sich nach langen Diskussionen auch schon von der alten Frakturschrift getrennt und war zur inzwischen fast allgemein üblichen Antiqua übergegangen. Wegen der Kostenvorteile des Stehsatzes konnte sie sich freilich in einzelnen Teilen noch sehr lange halten, etwa beim Schönfelder. Die Fraktur war die in Deutschland vom 16. bis zum Anfang des 2o. Jahrhunderts meistbenutzte Druckschrift, leicht gebrochen und mehr oder weniger verziert, anders als die sonst in Europa übliche Antiqua, die einfacher und klarer ist. Die Fraktur hatte für die 


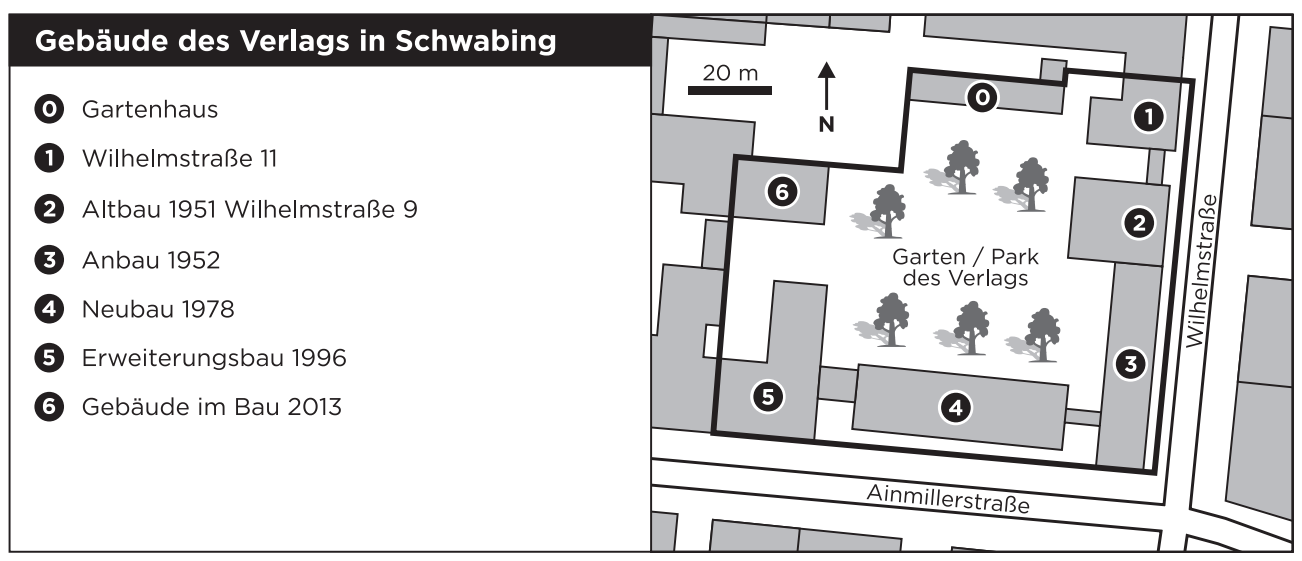

Lageplan der Verlagsgebäude.

juristischen, oft langen Texte von Gesetzen und Kommentaren den Vorteil, dass man mehr Worte auf einer Zeile unterbringen konnte, aber allgemein den Nachteil, dass es immer weniger Leser gab, die nach dem Krieg gewohnt waren, sie zu lesen. Das hatte politische Gründe. Als Adolf Hitler 1940/41 auf dem Höhepunkt seiner militärischen Erfolge davon träumte, ganz Europa vom Atlantik bis zum Ural zu erobern, hat er in einem Befehl von 194.1 die Verwendung der Fraktur verboten mit der Erklärung, sie sei gar keine deutsche Schrift, sondern eine «Schwabacher Judenletter», die so bezeichnet wurde, weil eine Zwischenform in der Entwicklung zur strengen Fraktur Schwabacher genannt wird und diese Bezeichnung angeblich auf einen Juden als Namensgeber zurückzuführen sei. Dahinter stand einfach der Wunsch, dass die anderen Europäer seine Propaganda in der ihnen vertrauten Antiqua lesen sollten, die deshalb Antiqua heißt, weil sie den einfachen großen Buchstaben der römischen Antike folgt. Fraktur kommt von lateinisch frangere, brechen, ist gebrochen und etwas verzwackt. 